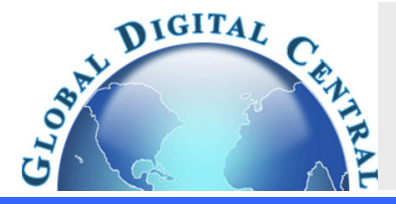

Frontiers in Heat and Mass Transfer

Available at www.ThermalFluidsCentral.org

\title{
ACCELERATING MHD FLOW OF A GENERALIZED OLDROYD-B FLUID WITH FRACTIONAL DERIVATIVE
}

\author{
Yaqing Liu ${ }^{\mathrm{a},}$ *, Jinyu Mab \\ ${ }^{a}$ Gengdan Institute of Beijing University of Technology, Beijing 101301, China \\ ${ }^{b}$ NCPC Hebei huamin pharmaceutical Co., Ltd, Shijiazhuang 052165, China
}

\begin{abstract}
This paper presents an exact solution for the magnetohydrodynamic (MHD) flow of an incompressible generalized Oldroyd-B fluid due to an infinite accelerating plate. The fractional calculus approach is introduced to establish the constitutive relationship of the Oldroyd-B fluid. The solutions in terms of Fox H-function are obtained by using the Laplace transform. When $N=0$ the solutions corresponds to the generalized Oldroyd-B fluids, while $\theta \rightarrow 0$ and $\lambda \rightarrow 0$ describes the Maxwell fluid and the generalized second fluid, as limiting cases of our general results, respectively.
\end{abstract}

Keywords: Generalized Oldroyd-B fluid; Exact solution; Laplace transform; Fox H-function.

\section{INTRODUCTION}

Non-Newtonian fluids are now considered to play a more important and appropriate role in industrial and technological applications. An important class of non-Newtonian fluids is viscoelastic fluids which exhibit both elastic and viscous property and many models of constitutive equations have been proposed to describe the transport behavior of these fluids. The Oldroyd-B fluid is a special viscoelastic non-Newtonian fluids, it has been especially applied to the problems of having small dimensionless relaxation and retardation times.

Recently, the fractional derivatives (Podlubny, 1999) are found to be quite flexible for describing the behaviors of viscoelastic fluids. Many researchers have studied different problems related to such fluids. In their works, the constitutive equations for generalized non-Newtonian fluids are modified from the well known fluid models by replacing the time derivative of an integer order by the so-called Riemann-Liouville fractional calculus operators. Haitao and $\mathrm{Xu}$ (2007) investigated the Stokes' problem for a viscoelastic fluid with a generalized Oldroyd-B model. Khan et al. (2009) and Hyder et al. (2009) considered some accelerated flows and due to pressure gradient flows of generalized Oldroyd-B fluid, respectively. Hyder (2009) discussed the flows of generalized Oldroyd-B fluid between two side walls perpendicular to the plate. Fetecau, Prasad and Rajagopal (2007), Con. Fetecau et al. (2009) and Vieru et al. (2008) investigated some accelerated flows of a generalized Oldroyd-B fluid. Hayat et al. $(2007,2009)$ studied the flow of a Maxwell fluid between two side walls. Khan et al. (2006) studied the MHD flow of a generalized Oldroyd-B fluid in a circular pipe.

Analytic solutions are important than numerical solutions because these are valid on the whole domain of definition whereas the numerical solutions are only valid at chosen points in the domain of definition. In this paper, we consider the MHD flow of an incompressible generalized Oldroyd-B fluid due to an infinite accelerating plate. The exact solutions for the velocity and shear stress fields are obtained by using the discrete Laplace transform technique for the fractional calculus. Moreover, the solutions for generalized Oldroyd-B fluid, fractional second grade fluid and fractional Maxwell fluid can be recovered by the current analysis.

\section{GOVERNING EQUATIONS}

We consider the MHD flow of an incompressible generalized OldroydB fluid due to an infinite accelerating plate. Assuming the velocity field and stress of the form

$$
V=u(y, t) \mathrm{i}, S=S(y, t)
$$

where $u$ is the velocity and $i$ is the unit vectors in the $x$-direction, taking account of the initial condition

$$
S(y, 0)=0, y>0
$$

the fluid being at rest up to the time $t=0$, we get

$\left(1+\lambda D_{t}^{\alpha}\right) S_{x y}=\mu\left(1+\lambda_{\mathrm{r}} D_{t}^{\beta}\right) \partial_{y} u(y, t)$

$S_{y y}=S_{z z}=S_{x z}=S_{y z}=0, S_{x y}=S_{y x}$. Consider that the conducting fluid is permeated by an imposed magnetic field $B_{0}$ which acts in the positive $y$ - coordinate. In the low- magnetic Reynolds number approximation, the magnetic body force is represented $\sigma B_{0}^{2} u$. Then, in the absence of a pressure gradient in the $x$-direction, the equation of motion yields the following scalar equations:

$$
\rho \frac{\partial u}{\partial t}=\frac{\partial}{\partial y} S_{x y}-\sigma B_{0}^{2} u
$$

\footnotetext{
* Corresponding author. Email: liuyaqing1981@163.com
} 
where $\rho$ is the constant density of the fluid. Eliminating $S_{x y}$ between Eq. (3) and Eq. (4), we arrive at the following fractional differential equation

$$
\left(1+\lambda D_{t}^{\alpha}\right) \frac{\partial u(y, t)}{\partial t}=v\left(1+\lambda_{\mathrm{r}} D_{t}^{\beta}\right) \frac{\partial^{2} u(y, t)}{\partial y^{2}}-M^{2}\left(1+\lambda D_{t}^{\alpha}\right) u(y, t)
$$

where $D_{t}^{\alpha}$ and $D_{t}^{\beta}$ are based on Riemann- Liouville's definition is defined as (Podlubny, 1999), $v=\mu / \rho$ is the kinematic viscosity and $M^{2}=\frac{\sigma B_{0}^{2}}{\rho}$. The associate initial and boundary conditions as follow:

Initial condition: $u(y, 0)=\frac{\partial u(y, 0)}{\partial t}=0, \quad y>0$

Boundary conditions: $u(0, t)=A t, t>0$

$u(y, t), \frac{\partial u(y, t)}{\partial y} \rightarrow 0$ as $y \rightarrow \infty, t>0$

where $u$ is velocity in the $x$-coordinate direction.

\section{EXACT SOLUTIONS}

Employing the non-dimensional quantities in Eqs. (5)-(8):

$u^{*}=\frac{u}{A}, y^{*}=\frac{y A \rho}{\mu}, t^{*}=\frac{t A^{2} \rho}{\mu}, \lambda^{*}=\lambda\left(\frac{A^{2} \rho}{\mu}\right)^{\alpha}, \lambda_{\mathrm{r}}^{*}=\lambda_{\mathrm{r}}\left(\frac{A^{2} \rho}{\mu}\right)^{\beta}$

Dimensionless motion and equations can be given (for brevity the dimensionless mark “*” is omitted here)

$\left(1+\lambda D_{t}^{\alpha}\right) \frac{\partial u(y, t)}{\partial t}=\left(1+\lambda_{\mathrm{r}} D_{t}^{\beta}\right) \frac{\partial^{2} u(y, t)}{\partial y^{2}}-M^{*}\left(1+\lambda D_{t}^{\alpha}\right) u(y, t)$

where $M^{*}=\frac{M^{2}}{\rho^{2} A^{2}}$. Using Laplace transforms principle of sequential fractional derivatives, yields

$$
\frac{\partial^{2} U(y, t)}{\partial y^{2}}-\frac{\left(p+M^{*}\right)\left(1+\lambda p^{\alpha}\right)}{\left(1+\lambda_{\mathrm{r}} p^{\beta}\right)} U(y, t)=0
$$

Boundary conditions: $U(0, t)=\frac{1}{p^{2}}$

$U(y, t), \frac{\partial U(y, t)}{\partial y} \rightarrow 0$ as $y \rightarrow \infty$

Solving Eqs. (11)- (13), one obtains

$$
U=\frac{1}{p^{2}} \exp \left[-\left(\frac{\left(p+M^{*}\right)\left(1+\lambda p^{\alpha}\right)}{1+\lambda_{\mathrm{r}} p^{\beta}}\right)^{\frac{1}{2}} y\right]
$$

The stress field can be calculated from Eq. (3). Taking the Laplace transform of Eq. (3) and introducing Eq. (14), obtain

$$
\bar{\tau}(y, p)=-\frac{1}{p^{2}}\left(p+M^{*}\right)^{\frac{1}{2}}\left(\frac{1+\lambda p^{\alpha}}{1+\lambda_{\mathrm{r}} p^{\beta}}\right)^{-\frac{1}{2}} \exp \left[-\left(\frac{\left(p+M^{*}\right)\left(1+\lambda p^{\alpha}\right)}{1+\lambda_{\mathrm{r}} p^{\beta}}\right)^{\frac{1}{2}} y\right]
$$

where $\bar{\tau}(y, p)$ is the Laplace transform of $\tau(y, t)$ and $\tau(y, t)=S_{x y} /\left(A^{2} \rho\right)$.
In order to avoid the burdensome calculations of residues and contour integrals, we will apply discrete inverse Laplace transform to get to the velocity and the stress fields. Writing Eq. (14) as series forms

$$
\begin{aligned}
& U(y, p)=\frac{1}{p^{2}}+\sum_{k=1}^{\infty} \frac{(-y)^{k}}{k !} \sum_{l=0}^{\infty} \frac{\left(-M^{*}\right)^{l}}{l !}\left(\frac{\lambda}{\lambda_{\mathrm{r}}}\right)^{\frac{k}{2}} \sum_{m=0}^{\infty} \frac{(-\lambda)^{m}}{m !} \sum_{n=0}^{\infty}\left(-\lambda_{\mathrm{r}}^{-1}\right)^{n} \\
& \times \frac{1}{n !} \frac{\Gamma\left(l-\frac{k}{2}\right)}{\Gamma\left(-\frac{k}{2}\right)} \frac{\Gamma\left(m-\frac{k}{2}\right)}{\Gamma\left(-\frac{k}{2}\right)} \frac{\Gamma\left(n+\frac{k}{2}\right)}{\Gamma\left(\frac{k}{2}\right)} \frac{1}{p^{\frac{k}{2}(\beta-\alpha-1)+\alpha m+\beta n+l+2}}
\end{aligned}
$$

Then, applying the inversion formulae term by term for the Laplace transform, yields

$$
\begin{aligned}
& u(y, t)=t+\sum_{k=1}^{\infty} \frac{(-y)^{k}}{k !} \sum_{l=0}^{\infty} \frac{\left(-M^{*}\right)^{l}}{l !}\left(\frac{\lambda}{\lambda_{\mathrm{r}}}\right)^{\frac{k}{2}} \sum_{m=0}^{\infty} \frac{(-\lambda)^{m}}{m !} \sum_{n=0}^{\infty}\left(-\lambda_{\mathrm{r}}^{-1} t^{\beta}\right)^{n} \frac{1}{n !} \\
& \times \frac{\Gamma\left(l-\frac{k}{2}\right)}{\Gamma\left(m-\frac{k}{2}\right)} \frac{\Gamma\left(n+\frac{k}{2}\right)}{\Gamma\left(-\frac{k}{2}\right)} \frac{t^{\frac{k}{2}(\beta-\alpha-1)+\alpha m+l+1}}{\Gamma\left(-\frac{k}{2}\right)} \frac{k\left(\frac{k}{2}\right)}{\Gamma\left(\frac{k}{2}(\beta-\alpha-1)+\alpha m+\beta n+l+2\right)} .
\end{aligned}
$$

In terms of Fox $\mathrm{H}$-function, we rewriting the above equation as the simpler form

$$
\begin{aligned}
& u(y, t)=t+\sum_{k=1}^{\infty} \frac{(-y)^{k}}{k !} \sum_{l=0}^{\infty} \frac{\left(-M^{*}\right)^{l}}{l !}\left(\frac{\lambda}{\lambda_{\mathrm{r}}}\right)^{\frac{k}{2}} \sum_{m=0}^{\infty} \frac{(-\lambda)^{m}}{m !} t^{\frac{k}{2}(\beta-\alpha-1)+\alpha m+l+1} \\
& \times H_{3,5}^{1,3}\left[\lambda_{\mathrm{r}}^{-1} t^{\beta} \mid \begin{array}{l}
\left(1-l+\frac{k}{2}, 0\right),\left(1-m+\frac{k}{2}, 0\right),\left(1-\frac{k}{2}, 1\right), \\
(0,1),\left(1+\frac{k}{2}, 0\right),\left(1+\frac{k}{2}, 0\right),\left(1-\frac{k}{2}, 0\right),\left(\frac{k}{2}(\alpha+1-\beta)-\alpha m-l-1, \beta\right)
\end{array}\right]
\end{aligned}
$$

where the property of the Fox H-function is

$$
\sum_{n=0}^{\infty} \frac{(-z)^{n} \Pi_{j=1}^{p} \Gamma\left(a_{j}+A_{j} n\right)}{n ! \Pi_{j=1}^{q} \Gamma\left(b_{j}+B_{j} n\right)}=H_{p, q+1}^{1, p}\left[z \mid \begin{array}{c}
\left(1-a_{1}, A_{1}\right), \cdots\left(1-a_{p}, A_{p}\right) \\
(0,1),\left(1-b_{1}, B_{1}\right), \cdots\left(1-b_{q}, B_{q}\right)
\end{array}\right]
$$

Similarly with the velocity field, we write Eq. (15) as the series form

$$
\begin{aligned}
& \bar{\tau}(y, p)=-\sum_{k=0}^{\infty} \frac{(-y)^{k}}{k !} \sum_{l=0}^{\infty}\left(-M^{*}\right)^{l} \frac{1}{l !}\left(\frac{\lambda}{\lambda_{\mathrm{r}}}\right)^{\frac{k-1}{2}} \sum_{m=0}^{\infty} \frac{(-\lambda)^{-m}}{m !} \\
& \times \sum_{n=0}^{\infty} \frac{\left(-\lambda_{\mathrm{r}}\right)^{-n}}{n !} \frac{\Gamma\left(l-\frac{k+1}{2}\right)}{\Gamma\left(-\frac{k+1}{2}\right)} \frac{\Gamma\left(m-\frac{k-1}{2}\right)}{\Gamma\left(-\frac{k-1}{2}\right)} \frac{\Gamma\left(n+\frac{k-1}{2}\right)}{\Gamma\left(\frac{k-1}{2}\right)} \\
& \times \frac{1}{p^{\frac{k-1}{2}(\beta-\alpha-1)+\alpha m+\beta n+l+1}}
\end{aligned}
$$

And using the discrete inverse Laplace transform, yields

$$
\tau(y, t)=-\sum_{k=0}^{\infty} \frac{(-y)^{k}}{k !} \sum_{l=0}^{\infty}\left(-M^{*}\right)^{l} \frac{1}{l !}\left(\frac{\lambda}{\lambda_{\mathrm{r}}}\right)^{\frac{k-1}{2}} \sum_{m=0}^{\infty} \frac{(-\lambda)^{-m}}{m !}
$$




$$
\begin{aligned}
& \times \sum_{n=0}^{\infty} \frac{\left(-\lambda_{\mathrm{r}}\right)^{-n}}{n !} \frac{\Gamma\left(l-\frac{k+1}{2}\right)}{\Gamma\left(-\frac{k+1}{2}\right)} \frac{\Gamma\left(m-\frac{k-1}{2}\right)}{\Gamma\left(-\frac{k-1}{2}\right)} \frac{\Gamma\left(n+\frac{k-1}{2}\right)}{\Gamma\left(\frac{k-1}{2}\right)} \\
& \times \frac{t^{\frac{k-1}{2}(\beta-\alpha-1)+\alpha m+\beta n+l}}{\Gamma\left(\frac{k-1}{2}(\beta-\alpha-1)+\alpha m+\beta n+l+1\right)}
\end{aligned}
$$

In terms of the Fox H-function, we obtain

$$
\begin{aligned}
& \tau(y, t)=-\sum_{k=0}^{\infty} \frac{(-y)^{k}}{k !} \sum_{l=0}^{\infty}\left(-M^{*}\right)^{l} \frac{1}{l !}\left(\frac{\lambda}{\lambda_{\mathrm{r}}}\right)^{\frac{k-1}{2}} \sum_{m=0}^{\infty} \frac{(-\lambda)^{-m}}{m !} t^{\frac{k-1}{2}(\beta-\alpha-1)+\alpha m+l} \\
& \times H_{3,5}^{1,3}\left[\lambda_{\mathrm{r}}^{-1} t^{\beta} \mid \begin{array}{l}
\left(1-l+\frac{k+1}{2}, 0\right),\left(1-m+\frac{k-1}{2}, 0\right),\left(1-\frac{k-1}{2}, 1\right), \\
(0,1),\left(1+\frac{k+1}{2}, 0\right),\left(1+\frac{k-1}{2}, 0\right),\left(1-\frac{k-1}{2}, 0\right),\left(\frac{k-1}{2}(\alpha+1-\beta)-\alpha m-l, \beta\right)
\end{array}\right]
\end{aligned}
$$

\section{SPECIAL CASES}

(i) When $M^{*}=0$, our solution reduced to the generalized Oldroyd-B fluid, as obtained by Khan et al. (2009). We attain to the velocity field

$u(y, t)=t+\sum_{k=1}^{\infty} \frac{(-y)^{k}}{k !}\left(\frac{\lambda}{\lambda_{\mathrm{r}}}\right)^{\frac{k}{2}} \sum_{m=0}^{\infty} \frac{(-\lambda)^{m}}{m !} t^{\frac{k}{2}(\beta-\alpha-1)+\alpha m+1}$

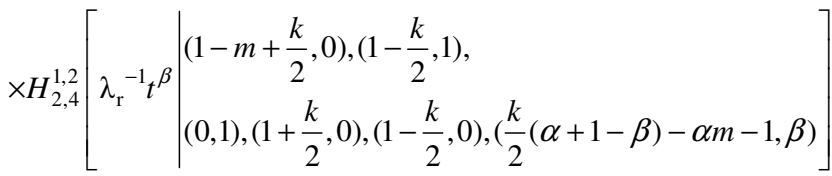

and the associated shear stress

$$
\begin{aligned}
& \tau(y, t)=-\sum_{k=0}^{\infty} \frac{(-y)^{k}}{k !}\left(\frac{\lambda}{\lambda_{\mathrm{r}}}\right)^{\frac{k-1}{2}} \sum_{m=0}^{\infty} \frac{(-\lambda)^{-m}}{m !} t^{\frac{k-1}{2}(\beta-\alpha-1)+\alpha m} \\
& \times H_{2,4}^{1,2}\left[\lambda_{\mathrm{r}}{ }^{-1} t^{\beta} \mid \begin{array}{l}
\left(1-m+\frac{k-1}{2}, 0\right),\left(1-\frac{k-1}{2}, 1\right), \\
(0,1),\left(1+\frac{k-1}{2}, 0\right),\left(1-\frac{k-1}{2}, 0\right),\left(\frac{k-1}{2}(\alpha+1-\beta)-\alpha m, \beta\right)
\end{array}\right]
\end{aligned}
$$

(ii) If $\alpha \neq 0, \lambda \rightarrow 0$, then Eqs.(18) and (22) can be simplified as

$$
\begin{aligned}
& u(y, t)=t+\sum_{k=1}^{\infty} \frac{(-y)^{k}}{k !} \sum_{l=0}^{\infty}\left(-M^{*}\right)^{l} \frac{1}{l !}\left(\frac{1}{\lambda_{\mathrm{r}}}\right)^{\frac{k}{2}} t^{\frac{k}{2}(\beta-1)+l+1}
\end{aligned}
$$

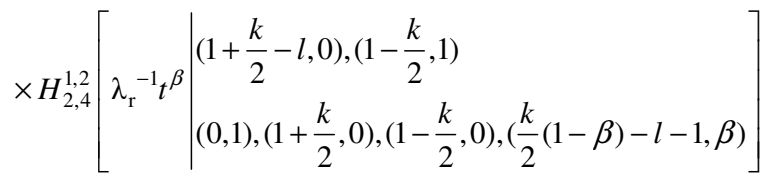

$$
\begin{aligned}
& \tau(y, t)=-\sum_{k=0}^{\infty} \frac{(-y)^{k}}{k !} \sum_{l=0}^{\infty}\left(-M^{*}\right)^{l} \frac{1}{l !}\left(\frac{1}{\lambda_{\mathrm{r}}}\right)^{\frac{k-1}{2}} t^{\frac{k-1}{2}(\beta-1)+l}
\end{aligned}
$$

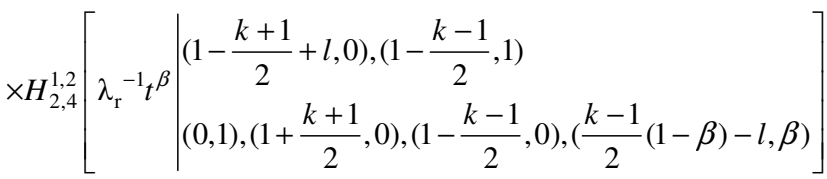

which represent the velocity and the stress fields for a generalized second grade fluid.

(iii) If $\beta \neq 0, \lambda_{\mathrm{r}} \rightarrow 0$, then Eqs. (19) and (22) can be simplified as

$$
\begin{aligned}
& \left.\begin{array}{l}
u(y, t)=t+\sum_{k=1}^{\infty} \frac{(-y)^{k}}{k !} \sum_{l=0}^{\infty} \frac{\left(-M^{*}\right)^{l}}{l !} \lambda^{\frac{k}{2}} t^{-\frac{k}{2}(\alpha+1)+l+1} \\
\times H_{2,4}^{1,2}\left[\lambda^{-1} t^{\alpha} \mid \begin{array}{l}
\left(1+\frac{k}{2}-l, 0\right),\left(1+\frac{k}{2}, 1\right) \\
(0,1),\left(1+\frac{k}{2}, 0\right),\left(1+\frac{k}{2}, 0\right),\left(\frac{k}{2}(\alpha+1)-l-1, \alpha\right)
\end{array}\right] \\
\tau(y, t)=-\sum_{k=0}^{\infty} \frac{(-y)^{k}}{k !} \sum_{l=0}^{\infty}\left(-M^{*}\right)^{l} \frac{1}{l !} \lambda^{\frac{k-1}{2}} t-\frac{k-1}{2}(\alpha+1)+l
\end{array}\right] \\
& \times H_{2,4}^{1,2}\left[\begin{array}{l}
\left.\lambda^{-1} t^{\alpha} \mid \begin{array}{l}
\left(1+\frac{k+1}{2}-l, 0\right),\left(1+\frac{k-1}{2}, 1\right) \\
(0,1),\left(1+\frac{k+1}{2}, 0\right),\left(1+\frac{k-1}{2}, 0\right),\left(\frac{k-1}{2}(\alpha+1)-l, \alpha\right)
\end{array}\right]
\end{array}\right.
\end{aligned}
$$

which correspond to the similar solutions for a generalized Maxwell fluid.

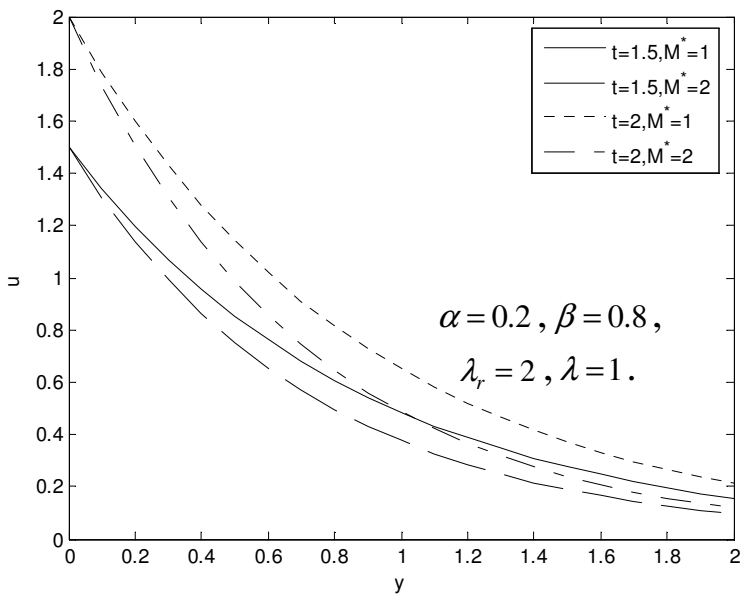

Fig. 1 Velocity fields for different values of $t$ and $M^{*}$ when keeping $\alpha$, $\beta, \lambda_{r}, \lambda$ fixed.

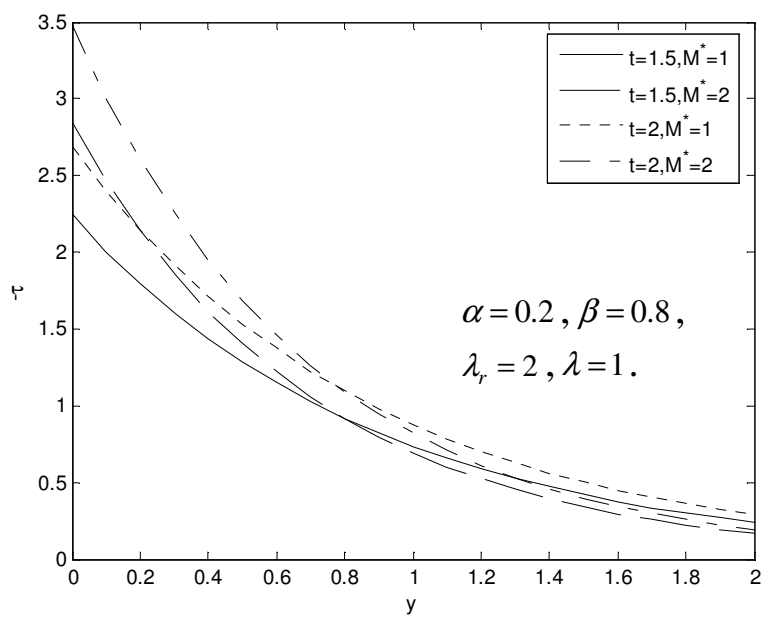

Fig. 2 Stress fields for different values of $t$ and $M^{*}$ when keeping $\alpha$, $\beta, \lambda_{r}, \lambda$ fixed. 


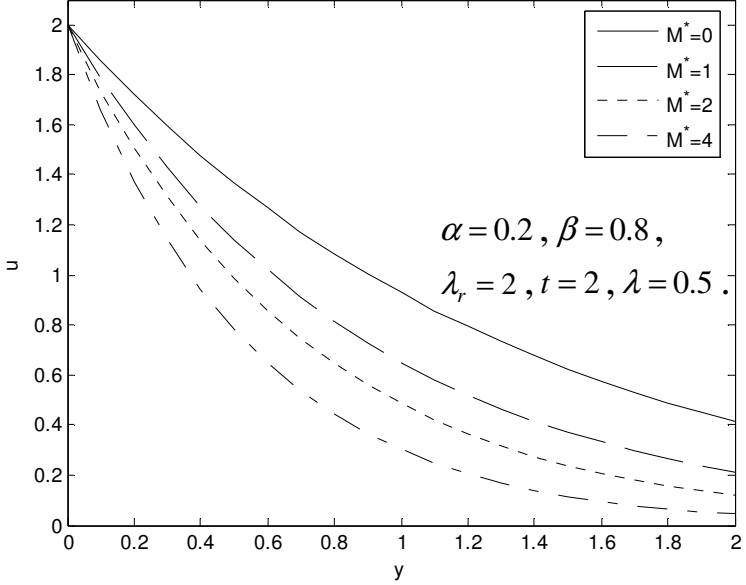

Fig. 3 Velocity fields for different values of $M^{*}$ when keeping $\alpha, \beta$, $\lambda_{r}, \lambda, t$ fixed.

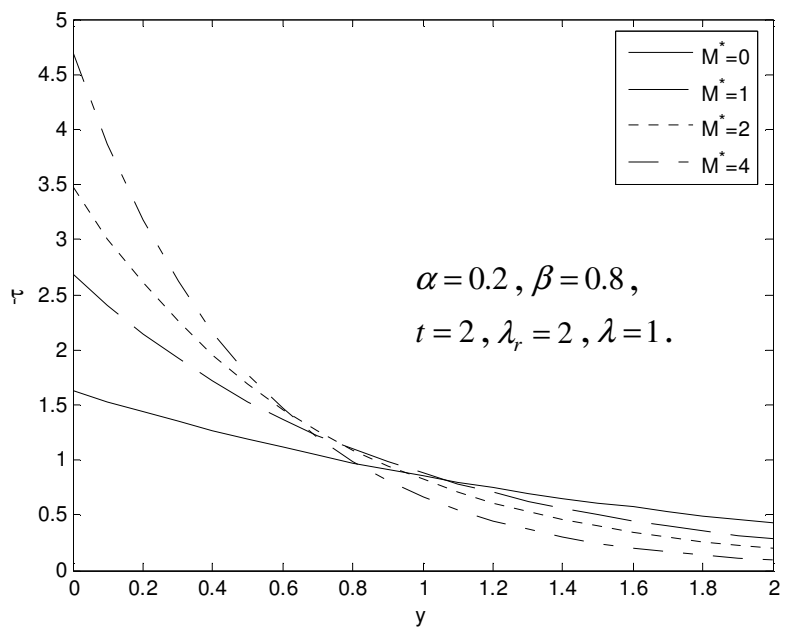

Fig. 4 Stress fields for different values of $M^{*}$ when keeping $\alpha, \beta, \lambda_{r}$, $\lambda, t$ fixed.

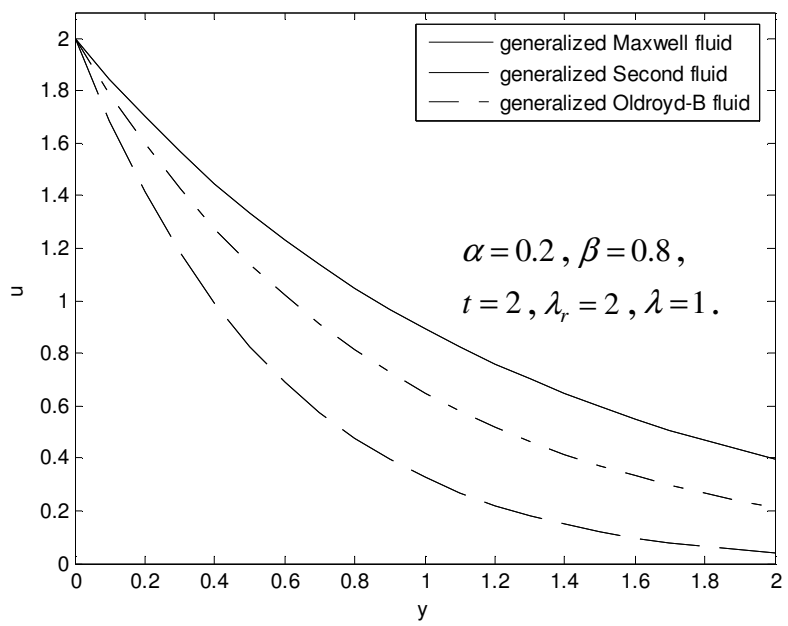

Fig. 5 Velocity fields for three types fluids when keeping $\alpha, \beta, \lambda_{r}, \lambda$, $t$ fixed.

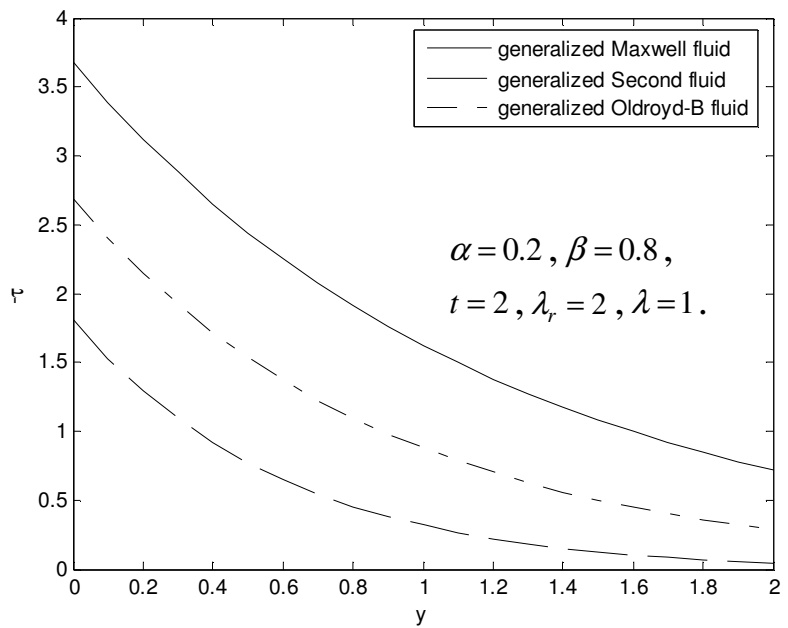

Fig. 6 Stress fields for three types fluids when keeping $\alpha, \beta, \lambda_{r}, \lambda, t$ fixed.

\section{NUMERICAL RESULTS AND DISCUSSION}

In this section, we analyze the characteristics of the velocity field and the stress fields by the presented figures. We can see from these figures that the magnetic body force is favorable to the velocity decays, and the more the values of $M^{*}$, the more rapidly the velocity decays.

Figures 1 and 2 indicate that the flow velocity as well as the shear stress increase with time or the magnetic body force increase. The strongest shear stress occurs near the plate and the shear stress decreases rapidly with the increase of distance from the plate. If $M^{*}=0$, the fluid not influenced by magnetic field. Figs. 3 and 4 demonstrate the influence of MHD on the velocity and the shear stress, respectively. Fig. 3 shows the smaller the values of $M^{*}$, the more slowly the velocity decays for the flow. Furthermore, with the increase in magnetic field the velocity decreases. Fig.4 shows the maximum stress occurs near the wall and decreases far away from the wall. Figs. 5-6 illustrate the velocity fields and shear stresses of the generalized Maxwell fluid, second grade fluid and Oldroyd-B fluid. From the figures, we can see that shear stress directly influence velocity field, the Maxwell fluid velocity is the fastest, the next is Oldroyd-B, and second grade fluid velocity is the slowest.

\section{CONCLUSIONS}

The purpose of this paper is to provide exact solutions for the unsteady flow of a generalized Oldroyd-B fluid due to an infinite accelerating plate. The exact solutions for the velocity and shear stress fields in terms of Fox $\mathrm{H}$-function are obtained by using the Laplace transform. Some previous and classical results, such as the velocity distribution for a viscous Maxwell, Oldroyd-B, and second grade fluid, can be considered as particular cases of our results. It is shown that the fractional constitutive relationship model is more useful than conventional model for describing the properties of viscoelastic fluid.

\section{ACKNOWLEDGEMENTS}

The work is supported by the Teaching Reform Project Foundations (No.GDJG201407) of BGD.

The authors wish to thank the Editor and Referees for their helpful comments and suggestions. 


\section{REFERENCES}

Fetecau C., Prasad Sharat C., Rajagopal K.R., 2007, "A Note on the Flow Induced by a Constantly Accelerating Plate in an Oldroyd-B Fluid," Applied Mathematical Modeling, 31, 647-654. http://dx.doi.org/10.1016/j.apm.2005.11.032

Fetecau Constantin, Fetecau Corina, Kamran M., Vieru D., 2009, "Exact Solutions for the Flow of a Generalized Oldroyd-B Fluid Induced by a Constantly Accelerating Plate Between Two Side Walls Perpendicular to the Plate," J. Non-Newtonian Fluid Mech, 156, 189-201. http://dx.doi.org/10.1016/j.jnnfm.2008.06.005

Hayat T., Sajid M., 2007, "Homotopy Analysis of MHD Boundary Layer Flow of an Upper-Convected Maxwell Fluid," International Journal of Engineering Science, 45, 393-401.

http://dx.doi.org/10.1016/j.ijengsci.2007.04.009

Hayat T., Abbas Z., Sajid M., 2009, "MHD Stagnation-Point Flow of an Upper-Convected Maxwell Fluid Over a Stretching Surface," Chaos, Solitons and Fractals, 39, 840-848.

http://dx.doi.org/10.1016/j.chaos.2007.01.067

Hyder Ali Muttaqi Shah S., Khan M., Qi Haitao, 2009, "Exact Solutions for a Viscoelastic Fluid with the Generalized Oldroyd-B Model," Nonlinear Analysis: Real World Applications, 10, 2590-2599. http://dx.doi.org/10.1016/j.nonrwa.2008.03.012
Hyder Ali Muttaqi Shah S., 2009, "Some Accelerated Flows of Generalized Oldroyd-B Fluid Between Two Side Walls Perpendicular to the Plate," Nonlinear Analysis: Real World Applications, 10, 2146-2150. http://dx.doi.org/10.1016/i.nonrwa.2008.03.023

Khan M., HayaT. t, Asghar S., 2006, "Exact Solution for MHD Flow of a Generalized Oldroyd-B Fluid with Modified Darcy's Law," International Journal of Engineering Science, 44, 333-339. http://dx.doi.org/10.1016/j.ijengsci.2005.12.004

Khan M., Hyder Ali S., Qi Haitao, 2009, "Some Accelerated Flows for a Generalized Oldroyd-B Fluid," Nonlinear Analysis: Real World Applications, 10, 980-991.

http://dx.doi.org/10.1016/j.nonrwa.2007.11.017

Podlubny I., 1999, Fractional Differential Equations. New York: Academic Press.

Qi H.T., Xu M.Y., 2007, “Stokes' First Problem for a Viscoelastic Fluid with the Generalized Oldroyd-B Model," Acta Mech. Sin., 23, 463-469. http://dx.doi.org/ 10.1007/s10409-007-0093-2

Vieru D., Fetecau Corina, Fetecau C., 2008, "Flow of a Generalized Oldroyd-B Fluid Due to a Constantly Accelerating Plate," Applied Mathematics and Computation, 201, 834-842. http://dx.doi.org/10.1016/j.amc.2007.12.045. 